\title{
Factors Affecting Vascular Clogging, Wound Status and Bacterial Culture in Diabetic Foot Ulcers
}

\author{
Jong-Lim Kim ${ }^{1,2}$, Jin Yong Shin ${ }^{1,2}$, Si-Gyun Roh ${ }^{1,2}$, Suk Choo Chang ${ }^{1,2}$, Nae-Ho Lee Le, $^{1,2}$ \\ ${ }^{1}$ Department of Plastic and Reconstructive Surgery, Chonbuk National University Medical School, Jeonju; ${ }^{2}$ Research Institute of Clinical Medicine-Biomedical \\ Research Institute of Chonbuk National University Hospital, Jeonju, Korea
}

\begin{abstract}
Background: We assumed that vascular clogging, wound status, and bacterial culture tests are significant characteristics in developing a treatment plan for diabetic patients with foot ulcers. The goal of our study was to find out the potential factors affecting vascular clogging, wound status, and bacterial culture tests in diabetic patients with foot ulcers.

Methods: The medical records of diabetic patients with foot ulcers presenting at our institution between January 2016 and December 2017 were reviewed. A total of 58 diabetic patients with foot ulcers were included. Patient baseline characteristics including co-morbidities, as well as foot and ulcer characteristics, were analyzed.

Results: By using the univariate and multivariate binary logistic regression analysis, we have found significant factors affecting vascular clogging, wound status and bacterial culture test. Age, glomerular filtration rate (GFR), and glycosylated hemoglobin ( $\mathrm{HbA1c}$ ) were significant factors influencing vascular status. Age, dialysis, GFR, white blood cell (WBC), and osteomyelitis were significant factors influencing wound status. Also, sex (male), dialysis and high WBC affect the possibility of Gram-negative aerobic bacterial infection more so than other bacterial infection.

Conclusion: This study has confirmed significant factors affecting vascular status, wound status, and bacterial culture tests, including co-morbidities, as well as foot and ulcer characteristics at baseline. Physicians should consider these factors in developing treatment plans or predicting outcomes of diabetic foot ulcers.
\end{abstract}

Keywords: Diabetic foot ulcer; Wound classification; Vascular clogging; Bacterial culture

\section{Introduction}

Diabetes is one of the leading causes of chronic disease and limb loss worldwide. It affected about 451 million (age of 18-99 years) people in 2017. By 2045, the number of reported diabetes cases are expected to increase to 693 million. Diabetic foot problems are common around the world, resulting in major difficulties in daily lives and also economic consequences for patients, their families, and society in general.

It is very important for clinicians to know which of the available clinical and laboratory findings are associated with poor outcomes in diabetic patients with foot ulcers. Prediction of outcomes in diabetic patients with foot ulcers might be helpful for clinicians to optimize and individualize management strategies.

In previous studies, peripheral artery diseases (PAD), presence of infection, and diabetic foot ulcer classification according to staging systems such as perfusion, extent, depth, infection and sensation (PEDIS)/Infectious Diseases Society of America (IDSA) classification grades or diabetic foot infection (DFI) wound scores have all been shown to be prognostic factors for diabetic foot disease [1-6]. Therefore, we assumed that vascular clogging, wound status, and bacterial culture tests are relevant in developing a treatment plan or predicting the prognosis of diabetic patients with foot ulcers.

\section{Original Article}

Received: June 19, 2019

Revised: July 27, 2019

Accepted: July 27, 2019

\section{Corresponding author:} Jin Yong Shin, M.D.

Department of Plastic and Reconstructive Surgery, Chonbuk National University Medical School, 20 Geonji-ro, Deokjin-gu, Jeonju 54907, Korea

Tel: +82-63-250-1860

Fax: +82-63-250-1866

E-mail: psjyshin@gmail.com

This is an Open Access article distributed under the terms of the Creative Commons Attribution Non-Commercial License (http://creativecommons.org/licenses/by-nc/4.0/) which permits unrestricted non-commercial use, distribution, and reproduction in any medium, provided the original work is properly cited.

(c) 2019 Korean Wound Management Society 
The goal of our study was to find out the potential factors affecting vascular clogging, wound status, and bacterial culture tests in diabetic patients with foot ulcers.

\section{Methods}

\section{Patients and data collection}

The study was approved by the Institutional Review Board of Chonbuk National University Hospital (IRB No. 2016-10-020002) and performed in accordance with the principles of the Declaration of Helsinki. The informed consent was waived. The medical records of diabetic patients with foot ulcers presenting at our institution between January 2016 and December 2017 were reviewed. Data on age, sex, history of smoking, duration of diabetes mellitus, diabetic foot ulcer classification (PEDIS/IDSA grade, and DFI wound score), wound angiosome, underlying disease (hypertension, congestive heart failure, dyslipidemia, chronic kidney disease [CKD] stage), major vascular disease, history of dialysis, bacterial culture results, and blood test results (including glycosylated hemoglobin [HbA1c], white blood cell [WBC], creatinine [Cr], glomerular filtration rate [GFR]) had been obtained on admission to the hospital.

The results of all tests and epidemiological characteristics were checked via the medical records. All patients were examined for wound status, vascular clogging, and pathologic culture on admission.

Each ulcer was classified using both the PEDIS/IDSA grade and the DFI wound score. The IDSA and the International Working Group on the Diabetic Foot developed a system for classifying diabetic foot wounds. The system is referred to by the acronym PEDIS, which is comprised of the first letters of perfusion, extent (size), depth (tissue loss), infection, and sensation (neuropathy). PEDIS grades are from 1 to 4 (uninfected, mild, moderate, and severe).

Lipsky et al. [7] developed a 10-item scoring system to measure outcomes in studies of various antimicrobial treatments for DFIs. The scoring system consists of a semi-quantitative assessment of the signs of inflammation, combined with measurements of both wound size and depth. Explicit definitions, which allow numerical scoring of wound parameters, are provided. Wound measurements consist of size, depth, and the amount of undermining. The10-item wound score ranges from 3 to 49 .

The ulcer site was documented and categorized as angiosome, including anterior tibial artery, posterior tibial artery, and multiple vessels.

Computed tomographic angiography of lower extremities is usually performed to check the vascular status. However, because of the risk of contrast-induced nephropathy, patients with severely decreased kidney function were tested by radiologists using color Doppler ultrasonography on lower extremity vessels. The results were classified as either normal or abnormal, noting whether there were clogs in single or multiple vessels. For patients with abnormal vascular status, percutaneous transluminal angiography was performed, and if needed, the clogged vessels were revascularized [8-11]. Diabetic foot ulcer was classified using the two systems, the PEDIS/IDSA grade and the DFI wound score, at the time of admission. Debridement was performed by removing all necrotic tissues. Bacterial culture was performed using the necrotic tissue obtained.

Three-phase bone scan was performed for suspected osteomyelitis (e.g., ulcer depth $>3 \mathrm{~mm}$, purulence in exposed bone of foot, or erythrocyte sedimentation rate $>60 \mathrm{~mm} / \mathrm{hr}$, etc.) at the time of admission. Bone biopsy culture, a quite invasive method to evaluate osteomyelitis, was sometimes used in the operating room, but we have not adopted bone biopsy culture as a routine method.

\section{Statistical analysis}

R language version 3.3.3 (R Foundation for Statistical Computing, Vienna, Austria) and T\&F program version 2.5 (YooJin BioSoft, Seoul, Korea) were used for all statistical analyses.

Binary logistic regression analysis was performed, and for analyzing the combined effect of more than two variables, multivariable logistic regression analysis was performed using the backward stepwise procedure as the variable selection method to minimize Akaike information criterion.

Univariate and multivariate binary logistic regressions were performed for PEDIS/IDSA grades (3, 4 vs. 1, 2), 10-item DFI wound score (cutoff, 20), vascular clogging (yes vs. no), and bacterial culture (others vs. Gram-aerobic).

\section{Results}

A total of 58 diabetic patients with foot ulcers were included. The characteristics of these patients are shown in Tables 1-3. The mean age of participants with ulcers was $60.5 \pm 13.4$ years. There were 45 men and 13 women. The mean duration of diabetes was $16.26 \pm 7.29$ years. The PEDIS/IDSA grade was 1 in 11 cases (19\%), 2 in 18 cases (31\%), 3 in 28 cases (48.3\%), and 
Table 1. Baseline characteristics $(n=58)$

\begin{tabular}{|c|c|}
\hline Variable & Value \\
\hline \multicolumn{2}{|l|}{ Sex } \\
\hline Female & $13(22.4)$ \\
\hline Male & $45(77.6)$ \\
\hline Age (yr) & $60.48 \pm 13.43$ \\
\hline \multicolumn{2}{|l|}{ PEDIS/IDSA grade } \\
\hline 1 & $11(19)$ \\
\hline 2 & $18(31)$ \\
\hline 3 & $28(48.3)$ \\
\hline 4 & $1(1.7)$ \\
\hline Wound parameters (10-item) & $7.0 \pm 4.5$ \\
\hline Wound parameters (8-item) & $4.7 \pm 3.1$ \\
\hline Wound measurements (subtotal) & $9.5 \pm 4.6$ \\
\hline DFI wound score (10-item) & $16.6 \pm 7.6$ \\
\hline DFI wound score (8-item) & $14.2 \pm 6.2$ \\
\hline \multicolumn{2}{|l|}{ Wound angiosome } \\
\hline ATA & $11(18.9)$ \\
\hline PTA & $19(32.8)$ \\
\hline Multi & $28(48.3)$ \\
\hline \multicolumn{2}{|l|}{ Wound angiosome } \\
\hline Single & $30(51.7)$ \\
\hline Multi & $28(48.3)$ \\
\hline \multicolumn{2}{|l|}{ Vascular clogging } \\
\hline No & $38(65.5)$ \\
\hline SFA & $4(6.9)$ \\
\hline PTA & $1(1.7)$ \\
\hline Multifocal artery & $15(25.9)$ \\
\hline \multicolumn{2}{|l|}{ Bacterial culture } \\
\hline Gram-positive aerobic & $22(37.9)$ \\
\hline Gram-positive \& -negative aerobic & $4(6.9)$ \\
\hline Gram-negative aerobic & $25(43.1)$ \\
\hline Others & $7(12.1)$ \\
\hline
\end{tabular}

Values are presented as number (\%) or mean \pm SD.

PEDIS, perfusion, extent, depth, infection and sensation; IDSA, Infectious Diseases Society of America; DFI, diabetic foot infection; ATA, anterior tibial artery; PTA, posterior tibial artery; SFA, superior femoral artery.

4 in one case (1.7\%). The mean DFI wound score (10-item) was $16.6 \pm 7.6$. With respect to vascular clogging, 38 (65.5\%) showed no clogging, five (8.6\%) showed single vessel blockage, and 15 (25.9\%) showed multi vessels blockage. Regarding bacterial cultures, 22 (37.9\%) showed Gram-positive aerobic, 25 (43.1\%) showed Gram-negative aerobic, four (6.9\%) showed Gram-positive \& negative aerobic, and seven (12.1\%) showed others.

Tables 4 and 5 show the univariate and multivariate binary logistic regression analyses for significant factors affecting vascular clogging in diabetic foot ulcer infection. According to the univariate analysis, age, Cr, and GFR were determined as significant factors affecting vascular status; whereas age, GFR, and $\mathrm{HbAlc}$ were found out to be significant factors influencing vascular status via multivariate analysis.

Tables 6-9 show the univariate and multivariate binary logistic regression analyses for the significant factors affecting diabetic foot ulcer classification in diabetic foot ulcer infection. In univariate analysis, WBC was the significant factor affecting diabetic foot ulcer classification (PEDIS/IDSA grade); whereas age, dialysis, GFR, WBC, and osteomyelitis were significant factors influencing wound status with diabetic foot ulcer classification in multivariate analysis (PEDIS/IDSA grade). Based on univariate analysis, osteomyelitis was the significant factor affecting diabetic foot ulcer classification (10-item DFI score); whereas in multivariate analysis, age, dialysis, GFR, WBC, and osteomyelitis were found out to be significant factors influencing wound status based on the wound classifications (10-item DFI score).

Tables 10 and 11 show the univariate and multivariate binary logistic regression analyses for the significant factors affecting bacterial culture (Gram-aerobic vs. others) in diabetic foot ulcer infection. According to the univariate analysis, low $\mathrm{HbAlc}$ affects the possibility of Gram-negative aerobic bacterial infection more than other bacterial infections, whereas in multivariate analysis, sex (male), dialysis, and high WBC affect the possibility of Gram-negative aerobic bacterial infection more than other bacterial infections.

\section{Discussion}

One of the most common complications of diabetes is the development of foot ulcers. Diabetic foot ulcers are related with serious morbidity as well as increased mortality. Therefore, understanding the factors that may contribute to the cure of diabetic foot ulcer would be helpful in both prevention and management strategies.

We have considered the factors affecting the treatment and prognosis of patients with diabetic foot ulcers as vascular clogging, current wound status, and the bacterial culture test, affecting antibiotic therapy. The severity and prediction of these three aspects can be helpful in predicting the prognosis of patients with diabetic foot ulcers and in determining the treat- 
Kim JL et al.

Factors in diabetic foot ulcers

Table 2. Baseline characteristics of patient history $(n=58)$

\begin{tabular}{|c|c|}
\hline Variable & Value \\
\hline \multicolumn{2}{|l|}{ Final status } \\
\hline No infection & $56(96.6)$ \\
\hline Hopeless & $1(1.7)$ \\
\hline Major amputation & $1(1.7)$ \\
\hline DM duration (yr) & $16.26 \pm 7.29$ \\
\hline \multicolumn{2}{|l|}{ Ulcer (history, n=25) } \\
\hline № & $2(8)$ \\
\hline Yes & $23(92)$ \\
\hline \multicolumn{2}{|c|}{ Antibiotic treatment (history, $n=24$ ) } \\
\hline No & $2(8.3)$ \\
\hline Yes & $22(91.7)$ \\
\hline \multicolumn{2}{|l|}{ Smoking } \\
\hline № & $34(58.6)$ \\
\hline Yes & $24(41.4)$ \\
\hline \multicolumn{2}{|l|}{ Hypertension } \\
\hline № & $24(41.4)$ \\
\hline Yes & $34(58.6)$ \\
\hline \multicolumn{2}{|l|}{ Retinopathy $(\mathrm{n}=33$ ) } \\
\hline № & $7(21.2)$ \\
\hline Yes & $26(78.8)$ \\
\hline \multicolumn{2}{|l|}{ Nephropathy } \\
\hline № & $31(53.4)$ \\
\hline Yes & $27(46.6)$ \\
\hline \multicolumn{2}{|l|}{ Neuropathy } \\
\hline № & $47(81)$ \\
\hline Yes & $11(19)$ \\
\hline \multicolumn{2}{|c|}{ IHD (coronary insufficiency) } \\
\hline № & $51(87.9)$ \\
\hline Yes & $7(12.1)$ \\
\hline \multicolumn{2}{|c|}{ Coronary artery angioplasty } \\
\hline № & $53(91.4)$ \\
\hline Yes & $5(8.6)$ \\
\hline \multicolumn{2}{|c|}{ Coronary artery bypass surgery } \\
\hline № & $57(98.3)$ \\
\hline Yes & $1(1.7)$ \\
\hline \multicolumn{2}{|c|}{ Congestive heart failure } \\
\hline № & $51(87.9)$ \\
\hline Yes & $7(12.1)$ \\
\hline \multicolumn{2}{|c|}{ Cerebrovascular disease } \\
\hline No & $57(98.3)$ \\
\hline Yes & $1(1.7)$ \\
\hline
\end{tabular}

Table 2. Continued

\begin{tabular}{lc}
\hline Variable & Value \\
\hline $\begin{array}{l}\text { Previous revascularization surgery (including PTA) } \\
\text { Dyslipidemia (n=2) }\end{array}$ & $9(100)$ \\
$\quad$ No & $1(50)$ \\
$\quad$ Yes & $1(50)$ \\
Dialysis & \\
No & $42(72.4)$ \\
Yes & $16(27.6)$ \\
CKD stage & \\
Not related & $31(53.4)$ \\
DDKT & $1(1.7)$ \\
Stage 2 & $1(1.7)$ \\
Stage 3 & $6(10.3)$ \\
Stage 3-4 & $1(1.7)$ \\
Stage 4 & $1(1.7)$ \\
Stage 4-5 & $1(1.7)$ \\
Stage 5 & $16(27.6)$ \\
\hline
\end{tabular}

Values are presented as number $(\%)$ or mean \pm SD.

DM, diabetes mellitus; IHD, ischemic heart disease; PTA, posterior tibial artery; CKD, chronic kidney disease; DDKT, death donor kidney transplantation.

Table 3. Baseline characteristics of blood test and osteomyelitis $(n=58)$

\begin{tabular}{lc}
\hline Variable & Value \\
\hline Creatinine $(\mathrm{mg} / \mathrm{dL})$ & $2.5 \pm 2.6$ \\
$\mathrm{GFR}(\mathrm{mL} / \mathrm{min})$ & $57.9 \pm 37.2$ \\
$\mathrm{Hb} 1 \mathrm{Ac}(\%)$ & $8.4 \pm 2.4$ \\
$\mathrm{WBC}\left(\times 10^{3} / \mathrm{\mu L}\right)$ & $10.7 \pm 5.1$ \\
Osteomyelitis & \\
$\quad$ No & $36(62.1)$ \\
Yes & $22(37.9)$ \\
\hline
\end{tabular}

Values are presented as mean $\pm S D$ or number (\%).

GFR, glomerular filtration rate; HbAlc, glycosylated hemoglobi; WBC, white blood cell.

ment strategy.

Recently, a number of larger studies reported the possible determinants of outcome in diabetic foot disease, such as the UK multicenter study initiated by Ince et al. [1] and the EURODIALE study [3]. These studies found that the presence of PAD was an independent predictor of the outcome. However, to the best of our knowledge, no previous studies had systematically-via multivariable analysis-assessed the potential factors that contribute to PAD, including co-morbidities, as well 
Kim JL et al.

Factors in diabetic foot ulcers

Table 4. Univariate binary logistic regression analysis using vascular clogging (yes vs. no)

\begin{tabular}{|c|c|c|c|}
\hline Predictor & Subgroup & OR (95\% Cl) & P-value \\
\hline Sex & Male vs. female & $2.024(0.487-8.407)$ & 0.332 \\
\hline Age & & $1.062(1.012-1.114)$ & $0.013^{\mathrm{a})}$ \\
\hline \multirow[t]{4}{*}{ PEDIS/IDSA grade (1-4) } & 2 vs.1 & $2.667(0.529-13.433)$ & 0.234 \\
\hline & 3 vs.1 & $1.067(0.224-5.075)$ & 0.935 \\
\hline & 4 vs.1 & 0 (0-infinity) & 0.992 \\
\hline & (3 or 4 ) vs. (1 or 2 ) & $0.540(0.180-1.621)$ & 0.272 \\
\hline Wound parameters (10-item) & & $0.958(0.848-1.083)$ & 0.497 \\
\hline DFI wound score (10-item) & & $0.976(0.907-1.049)$ & 0.506 \\
\hline 10-Item DFI score (cutoff =20) & $\geq 20$ vs. $<20$ & $0.458(0.138-1.521)$ & 0.202 \\
\hline \multirow[t]{2}{*}{ Wound angiosome (ATA, PTA, multi) } & PTA vs. ATA & $2.625(0.437-15.777)$ & 0.464 \\
\hline & Multi vs. ATA & $2.191(0.527-16.093)$ & 0.45 \\
\hline Wound angiosome & Multi vs. single & $1.510(0.508-4.484)$ & 0.458 \\
\hline \multirow[t]{2}{*}{ Bacterial culture } & Others vs. Gram-positive aerobic & $1.211(0.393-3.734)$ & 0.505 \\
\hline & Others vs. Gram-negative aerobic & $1.214(0.404-3.647)$ & 0.729 \\
\hline DM duration & & $1.025(0.951-1.104)$ & 0.522 \\
\hline Ulcer (history) & Yes vs. no & $0.150(0.007-3.092)$ & 0.219 \\
\hline Antibiotic treatment (history) & Yes vs. no & $0.222(0.011-4.358)$ & 0.322 \\
\hline Smoking & Yes vs. no & $0.917(0.304-2.760)$ & 0.877 \\
\hline Hypertension & Yes vs. no & $3.000(0.908-9.914)$ & 0.072 \\
\hline Retinopathy & Yes vs. no & $1.111(0.177-6.991)$ & 0.911 \\
\hline Nephropathy & Yes vs. no & $2.300(0.761-6.952)$ & 0.14 \\
\hline Neuropathy & Yes vs. no & $0.662(0.155-2.833)$ & 0.578 \\
\hline IHD (coronary insufficiency) & Yes vs. no & $1.500(0.301-7.476)$ & 0.621 \\
\hline Coronary artery angioplasty & Yes vs. no & $1.296(0.198-8.473)$ & 0.786 \\
\hline Congestive heart failure & Yes vs. no & $2.917(0.583-14.586)$ & 0.192 \\
\hline Cerebrovascular disease & Yes vs. no & 0 (0-infinity) & 0.992 \\
\hline Dyslipidemia & Yes vs. no & 1.000 (0-infinity) & 1 \\
\hline Dialysis & Yes vs. no & $2.500(0.763-8.192)$ & 0.13 \\
\hline Creatinine & & $1.254(1.010-1.558)$ & $0.041^{\text {a) }}$ \\
\hline GFR & & $0.973(0.956-0.991)$ & $0.003^{b)}$ \\
\hline $\mathrm{Hb} 1 \mathrm{Ac}$ & & $0.935(0.723-1.209)$ & 0.608 \\
\hline WBC & & $0.969(0.866-1.084)$ & 0.576 \\
\hline Osteomyelitis & Yes vs. no & $0.826(0.268-2.545)$ & 0.739 \\
\hline
\end{tabular}

OR, odds ratio; $\mathrm{Cl}$, confidence interval; PEDIS, perfusion, extent, depth, infection and sensation; IDSA, Infectious Diseases Society of America; DFI, diabetic foot infection; ATA, anterior tibial artery; PTA, posterior tibial artery; DM, diabetes mellitus; IHD, ischemic heart disease; GFR, glomerular filtration rate; HbAlc, glycosylated hemoglobin; WBC, white blood cell.

a) $P<0.05 ;{ }^{\text {b) }} \mathrm{P}<0.01$.

as foot and ulcer characteristics at baseline. In our analysis, older age, decreased GFR, and HbAlc were significant factors contributing to the presence of PAD in diabetic foot ulcers patients (Fig. 1).

Aging is a significant predictor of the risk of limb amputa- tion and hospital mortality in diabetic patients with foot ulcers [12]. Aged patients usually have progressive atherosclerosis with peripheral neuropathy, $\mathrm{CKD}$, as well as PAD [13-15]. Our study has revealed that older agepatients have greater PAD among diabetic foot ulcers patients. 
Kim JL et al.

Factors in diabetic foot ulcers

Table 5. Multivariate binary logistic regression analysis using vascular clogging (yes vs. no)

\begin{tabular}{lll}
\hline Predictor & \multicolumn{1}{c}{ OR $(95 \% \mathrm{Cl})$} & P-value \\
\hline Age & $1.063(1.001-1.129)$ & $0.048^{\mathrm{a})}$ \\
GFR & $0.962(0.937-0.988)$ & $0.004^{\mathrm{b})}$ \\
Hb1Ac & $1.356(0.887-2.074)$ & 0.16
\end{tabular}

OR, odds ratio; $\mathrm{Cl}$, confidence interval; GFR, glomerular filtration rate; $\mathrm{HbAlc}$, glycosylated hemoglobin.

a) $\mathrm{P}<0.05$; $\mathrm{b} P<0.01$.
The etiologies of CKD and diabetic foot ulcers have many common pathogenetic pathways, with both etiologies involving vasculopathy and microangiopathy. In the kidney, the pathogenic pathways lead to endothelial dysfunction, activation of Ras and transforming growth factor- $\beta$, inflammation with podocytes, and mesangial cell death. All paths ultimately lead to renal vasoconstriction, fibrosis that leads to glomerulosclerosis, and tubular degeneration, resulting in a decrease in renal blood flow, GFR, and renal function. Several studies have

Table 6. Univariate binary logistic regression analysis using PEDIS/IDSA grade (3, 4 vs. 1, 2)

\begin{tabular}{|c|c|c|c|}
\hline Predictor & Subgroup & OR (95\% Cl) & P-value \\
\hline Sex & Male vs. female & $0.820(0.238-2.825)$ & 0.753 \\
\hline Age & & $1.020(0.981-1.061)$ & 0.318 \\
\hline \multirow[t]{5}{*}{ Vascular clogging } & SFA vs. no & $0.270(0.026-2.835)$ & 0.275 \\
\hline & PTA vs. no & 0 (0-infinity) & 0.991 \\
\hline & SFA or PTA vs. no & $0.202(0.021-1.984)$ & 0.17 \\
\hline & Multifocal artery vs. no & $0.708(0.214-2.35)$ & 0.573 \\
\hline & Yes vs. no & $0.540(0.180-1.621)$ & 0.272 \\
\hline \multirow[t]{2}{*}{ Bacterial culture } & Others vs. Gram-positive aerobic & $1.341(0.463-3.887)$ & 0.589 \\
\hline & Others vs. Gram-negative aerobic & $0.869(0.307-2.458)$ & 0.791 \\
\hline DM duration (yr) & & $1.003(0.934-1.077)$ & 0.928 \\
\hline Ulcer (history) & Yes vs. no & $1.300(0.072-23.435)$ & 0.859 \\
\hline Antibiotic treatment (history) & Yes vs. no & $1.444(0.080-26.231)$ & 0.804 \\
\hline Smoking & Yes vs. no & $0.752(0.264-2.145)$ & 0.594 \\
\hline Hypertension & Yes vs. no & $1.773(0.616-5.102)$ & 0.288 \\
\hline Retinopathy & Yes vs. no & $0.469(0.086-2.547)$ & 0.38 \\
\hline Nephropathy & Yes vs. no & $1.149(0.409-3.226)$ & 0.792 \\
\hline Neuropathy & Yes vs. no & $0.503(0.130-1.951)$ & 0.32 \\
\hline IHD (coronary insufficiency) & Yes vs. no & $0.721(0.146-3.552)$ & 0.688 \\
\hline Coronary artery angioplasty & Yes vs. no & $4.480(0.469-42.792)$ & 0.193 \\
\hline Coronary artery bypass surgery & Yes vs. no & 0 (0-infinity) & 0.991 \\
\hline Congestive heart failure & Yes vs. no & 0 (0-infinity) & 0.99 \\
\hline Previous revascularization surgery (including PTA) & Yes vs. no & 0 (0-infinity) & 0.996 \\
\hline Dyslipidemia & Yes vs. no & 0 (0-infinity) & 1 \\
\hline Dialysis & Yes vs. no & $2.018(0.620-6.569)$ & 0.244 \\
\hline Creatinine & & $1.064(0.867-1.305)$ & 0.553 \\
\hline GFR & & $1.005(0.990-1.019)$ & 0.533 \\
\hline $\mathrm{Hb} 1 \mathrm{Ac}$ & & $1.097(0.862-1.395)$ & 0.451 \\
\hline WBC & & $1.198(1.041-1.378)$ & $0.011^{\mathrm{a})}$ \\
\hline Osteomyelitis & Yes vs. no & $0.746(0.257-2.161)$ & 0.589 \\
\hline
\end{tabular}

PEDIS, perfusion, extent, depth, infection and sensation; IDSA, Infectious Diseases Society of America; OR, odds ratio; Cl, confidence interval; SFA, superior femoral artery; PTA, posterior tibial artery; DM, diabetes mellitus; IHD, ischemic heart disease; GFR, glomerular filtration rate; HbAlc, glycosylated hemoglobin; WBC, white blood cell.

a) $\mathrm{P}<0.05$ 
Kim JL et al.

Factors in diabetic foot ulcers

Table 7. Multivariate binary logistic regression analysis using PEDIS/IDSA grade (3, 4 vs. 1, 2)

\begin{tabular}{lcc}
\hline Predictor & OR $(95 \% \mathrm{Cl})$ & P-value \\
\hline Age & $1.061(1.004-1.122)$ & $0.037^{\mathrm{a})}$ \\
Dialysis (yes vs. no) & $21.370(1.961-232.917)$ & $0.012^{\mathrm{a})}$ \\
GFR & $1.031(1.001-1.062)$ & $0.042^{\mathrm{a})}$ \\
WBC & $1.245(1.054-1.470)$ & $0.01^{\mathrm{b}}$ \\
Osteomyelitis (yes vs. no) & $2.397(0.582-9.867)$ & 0.226 \\
\hline
\end{tabular}

PEDIS, perfusion, extent, depth, infection and sensation; IDAS, Infectious Diseases Society of America; OR, odds ratio; $\mathrm{Cl}$, confidence interval; GFR, glomerular filtration rate; WBC, white blood cell.

a) $\mathrm{P}<0.05$; ${ }^{\text {b) }}<0.01$. found that coexistence of CKD is associated with a greater severity of PAD [16-20]. In line with previous studies, our study has shown that decreased GFR was a significant factor predicting the presence of $\mathrm{PAD}$ in diabetic foot ulcers patients.

The measurement of the long-term blood glucose control index, $\mathrm{HbAlc}$, is used to evaluate and plan the treatment of diabetic patients. Chronic hyperglycemia is a well-known risk factor for diabetes-related vascular complications. Several studies have suggested that $\mathrm{HbAlc}$ may also be related with the incidence of large-vessel disease in patients with diabetes [21-24]. Our study was also consistent with the results of previous studies. In our study, $\mathrm{HbAlc}$ was a significant factor pre-

Table 8. Univariate binary logistic regression analysis using 10-item DFI score (cutoff $=20$ )

\begin{tabular}{|c|c|c|c|}
\hline Predictor & Subgroup & OR $(95 \% \mathrm{Cl})$ & P-value \\
\hline Sex & Male vs. female & $0.883(0.247-3.154)$ & 0.848 \\
\hline Age & & $1.025(0.984-1.069)$ & 0.24 \\
\hline \multirow[t]{5}{*}{ Vascular clogging } & SFA vs. no & 0 (0-infinity) & 0.993 \\
\hline & PTA vs. no & 0 (0-infinity) & 0.997 \\
\hline & SFA or PTA vs. no & 0 (0-infinity) & 0.992 \\
\hline & Multifocal artery vs. no & $0.687(0.197-2.404)$ & 0.557 \\
\hline & Yes vs. no & $0.458(0.138-1.521)$ & 0.202 \\
\hline \multirow[t]{2}{*}{ Culture } & Others vs. Gram-positive aerobic & $0.528(0.176-1.584)$ & 0.255 \\
\hline & Others vs. Gram-negative aerobic & $1.381(0.463-4.119)$ & 0.562 \\
\hline DM duration & & $0.984(0.912-1.060)$ & 0.666 \\
\hline Ulcer (history) & Yes vs. no & $0.643(0.036-11.632)$ & 0.765 \\
\hline Antibiotic treatment (history) & Yes vs. no & $0.692(0.038-12.572)$ & 0.804 \\
\hline Smoking & Yes vs. no & $1.494(0.505-4.415)$ & 0.468 \\
\hline Hypertension & Yes vs. no & $0.909(0.307-2.691)$ & 0.863 \\
\hline Retinopathy & Yes vs. no & $0.400(0.069-2.309)$ & 0.306 \\
\hline Nephropathy & Yes vs. no & $1.070(0.366-3.128)$ & 0.902 \\
\hline Neuropathy & Yes vs. no & $0.604(0.142-2.579)$ & 0.496 \\
\hline IHD (coronary insufficiency) & Yes vs. no & $1.375(0.277-6.833)$ & 0.697 \\
\hline Coronary artery angioplasty & Yes vs. no & $1.193(0.183-7.781)$ & 0.854 \\
\hline Coronary artery bypass surgery & Yes vs. no & 0 (0-infinity) & 0.992 \\
\hline Congestive heart failure & Yes vs. no & 0 (0-infinity) & 0.991 \\
\hline Dyslipidemia & Yes vs. no & 0 (0-infinity) & 1 \\
\hline Dialysis & Yes vs. no & $1.556(0.479-5.051)$ & 0.462 \\
\hline Creatinine & & $1.027(0.833-1.265)$ & 0.805 \\
\hline GFR & & $1.000(0.985-1.015)$ & 0.992 \\
\hline $\mathrm{Hb} 1 \mathrm{Ac}$ & & 1.147 (0.895-1.469) & 0.278 \\
\hline WBC & & $1.097(0.984-1.223)$ & 0.095 \\
\hline Osteomyelitis & Yes vs. no & $3.600(1.165-11.127)$ & $0.026^{a)}$ \\
\hline
\end{tabular}

DFI, diabetic foot infection; OR, odds ratio; Cl, confidence interval; SFA, superior femoral artery; PTA, posterior tibial artery; DM, diabetes mellitus; IHD, ischemic heart disease; GFR, glomerular filtration rate; HbAlc, glycosylated hemoglobin; WBC, white blood cell.

a) $\mathrm{P}<0.05$. 
Table 9. Multivariate binary logistic regression analysis using 10item DFI score (cutoff $=20$ )

\begin{tabular}{lcc}
\hline Predictor & OR $(95 \% \mathrm{Cl})$ & P-value \\
\hline Age & $1.067(1.002-1.136)$ & $0.044^{\mathrm{a})}$ \\
Dialysis (yes vs. no) & $18.288(1.323-252.735)$ & $0.03^{\mathrm{a})}$ \\
GFR & $1.030(0.994-1.068)$ & 0.106 \\
WBC & $1.203(1.033-1.401)$ & $0.017^{\mathrm{a})}$ \\
Osteomyelitis (yes vs. no) & $18.093(3.150-113.750)$ & $0.001^{\mathrm{b})}$ \\
\hline
\end{tabular}

DFI, diabetic foot infection; OR, odds ratio; $\mathrm{Cl}$, confidence interval; GFR, glomerular filtration rate; WBC, white blood cell.

a) $\mathrm{P}<0.05$; ${ }^{\text {b) }} \mathrm{P}<0.01$. dicting the presence of PAD in diabetic foot ulcers patients.

Wound classification systems help physicians to evaluate the prognosis of diabetic foot patients or to plan treatment plans. In our study, older age, dialysis patients, decreased GFR, high WBC, and concomitance of osteomyelitis were significant factors for determining the severity of foot disease (Fig. 2).

As mentioned earlier, elderly patients may have various complications, including PAD, which can have a negative impact on wound status. Decreased GFR or the need of dialysis may indicate severe progression of diabetes in patients, contributing to worsened wound status. Diabetic foot ulcers pa-

Table 10. Univariate binary logistic regression analysis using bacterial culture (Gram-aerobic vs. others)

\begin{tabular}{|c|c|c|c|}
\hline Predictor & Subgroup & $\mathrm{OR}(95 \% \mathrm{Cl})$ & P-value \\
\hline Sex & Male vs. female & $3.188(0.773-13.144)$ & 0.109 \\
\hline Age & & $0.995(0.957-1.034)$ & 0.795 \\
\hline \multirow{4}{*}{ PEDIS/IDSA grade (1-4) } & 2 vs. 1 & $1.400(0.300-6.535)$ & 0.669 \\
\hline & 3 vs. 1 & $1.517(0.361-6.373)$ & 0.57 \\
\hline & 4 vs. 1 & 0 (0-infinity) & 0.992 \\
\hline & 3 or 4 vs. 1 or 2 & $1.151(0.407-3.257)$ & 0.791 \\
\hline Wound parameters (10-item) & & $1.051(0.934-1.183)$ & 0.407 \\
\hline DFI wound score (10-item) & & $1.023(0.954-1.097)$ & 0.527 \\
\hline 10-Item DFI score (cutoff=20) & $\geq 20$ vs. $<20$ & $0.724(0.243-2.159)$ & 0.562 \\
\hline Wound angiosome (ATA, PTA, multi) & PTA vs. ATA & $0.750(0.169-3.327)$ & 0.705 \\
\hline Wound angiosome (ATA, PTA, multi) & Multi vs. ATA & $0.463(0.112-1.908)$ & 0.287 \\
\hline Wound angiosome & Multi vs. single & $0.556(0.194-1.593)$ & 0.274 \\
\hline \multirow[t]{5}{*}{ Vascular clogging } & SFA vs. no & 0 (0-infinity) & 0.993 \\
\hline & PTA vs. no & 0 (0-infinity) & 0.996 \\
\hline & SFA or PTA vs. no & 0 (0-infinity) & 0.992 \\
\hline & Multifocal artery vs. no & $1.412(0.426-4.683)$ & 0.573 \\
\hline & Yes vs. no & $0.824(0.274-2.473)$ & 0.729 \\
\hline DM duration (yr) & & $1.021(0.950-1.097)$ & 0.57 \\
\hline Smoking & Yes vs. no & $0.500(0.169-1.476)$ & 0.21 \\
\hline Hypertension & Yes vs. no & $1.105(0.384-3.180)$ & 0.853 \\
\hline Retinopathy & Yes vs. no & $6.000(0.631-57.058)$ & 0.119 \\
\hline Nephropathy & Yes vs. no & $1.470(0.517-4.181)$ & 0.47 \\
\hline Neuropathy & Yes vs. no & $1.125(0.300-4.212)$ & 0.861 \\
\hline IHD (coronary insufficiency) & Yes vs. no & $0.989(0.200-4.878)$ & 0.989 \\
\hline Coronary artery angioplasty & Yes vs. no & $0.302(0.032-2.887)$ & 0.299 \\
\hline Congestive heart failure & Yes vs. no & $1.905(0.386-9.411)$ & 0.429 \\
\hline Dyslipidemia & Yes vs. no & 1.000 (0-infinity) & 1 \\
\hline Dialysis & Yes vs. no & $3.000(0.910-9.889)$ & 0.071 \\
\hline
\end{tabular}


Kim JL et al.

Factors in diabetic foot ulcers

Table 10. Continued

\begin{tabular}{|c|c|c|c|}
\hline Predictor & Subgroup & OR $(95 \% \mathrm{Cl})$ & P-value \\
\hline \multirow[t]{6}{*}{ CKD stage } & DDKT vs. not related & 0 (0-infinity) & 0.997 \\
\hline & Stage 2 vs. not related & 0 (0-infinity) & 0.997 \\
\hline & Stage 3 vs. not related & $0.792(0.125-5.009)$ & 0.804 \\
\hline & Stage 3-4 vs. not related & 0 (0-infinity) & 0.997 \\
\hline & Stage 4-5 vs. not related & 0 (0-infinity) & 0.997 \\
\hline & Stage 5 vs. not related & $2.639(0.761-9.153)$ & 0.126 \\
\hline Creatinine & & $1.163(0.942-1.436)$ & 0.161 \\
\hline GFR & & $0.995(0.981-1.010)$ & 0.526 \\
\hline $\mathrm{Hb} 1 \mathrm{Ac}$ & & $0.695(0.510-0.947)$ & $0.021^{\mathrm{a})}$ \\
\hline WBC & & $1.065(0.958-1.184)$ & 0.24 \\
\hline Osteomyelitis & Yes vs. no & $0.336(0.107-1.053)$ & 0.061 \\
\hline
\end{tabular}

OR, odds ratio; Cl, confidence interval; PEDIS, perfusion, extent, depth, infection and sensation; IDSA, Infectious Diseases Society of America; DFI, diabetic foot infection; ATA, anterior tibial artery; PTA, posterior tibial artery; SFA, superior femoral artery; DM, diabetes mellitus; IHD, ischemic heart disease; CKD, chronic kidney disease; DDKT, death donor kidney transplantation; GFR, glomerular filtration rate; HbA1c, glycosylated hemoglobin; WBC, white blood cell.

a) $P<0.05$.

Table 11. Multivariate binary logistic regression analysis using bacterial culture (Gram-aerobic vs. others)

\begin{tabular}{lcc}
\hline Predictor & OR $(95 \%$ Cl) & P-value \\
\hline Sex (male vs. female) & $13.680(1.294-144.622)$ & $0.03^{\text {a) }}$ \\
Smoking (yes vs. no) & $0.071(0.011-0.464)$ & $0.006^{\text {b) }}$ \\
Dialysis (yes vs. no) & $3.868(0.644-23.234)$ & 0.139 \\
Hb1Ac & $0.525(0.320-0.862)$ & $0.011^{\text {a) }}$ \\
WBC & $1.271(1.062-1.522)$ & $0.009^{\text {b) }}$
\end{tabular}

OR, odds ratio; $\mathrm{Cl}$, confidence interval; HbAlc, glycosylated hemoglobi; WBC, white blood cell.

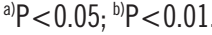

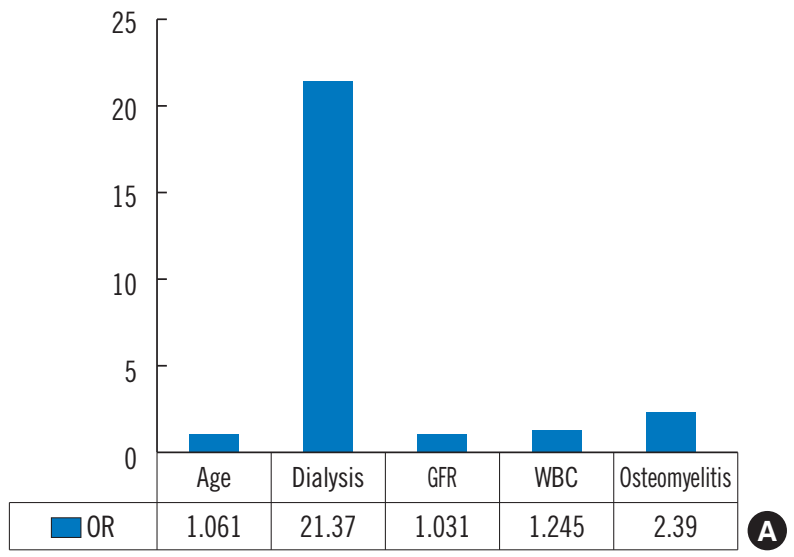

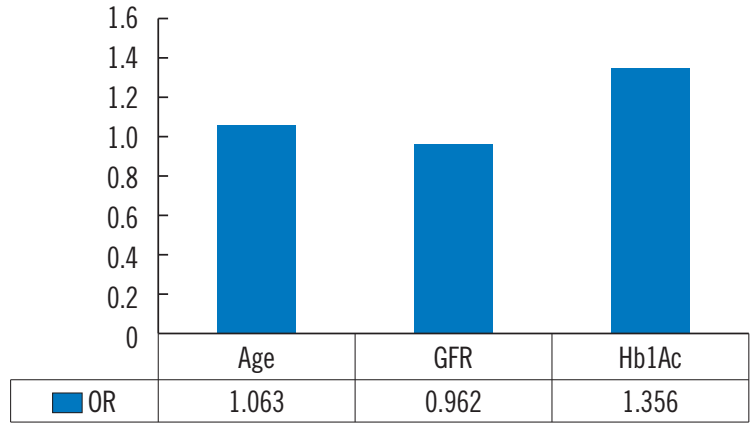

Fig. 1. Vascular clogging (yes vs. no) in DFU. Multivariate binary logistic regressing. DFU, diabetic foot ulcers; GFR, glomerular filtration rate; $\mathrm{HbAlc}$, glycosylated hemoglobin; $\mathrm{OR}$, odds ratio.

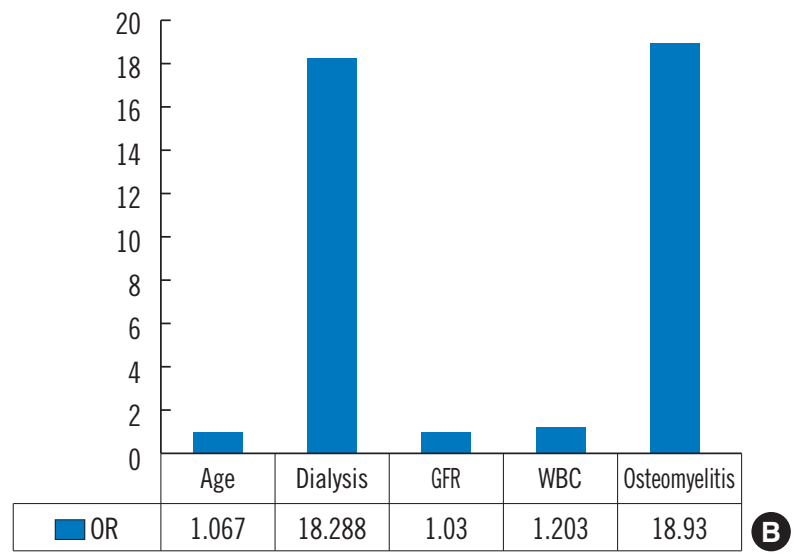

Fig. 2. Multivariate binary logistic regressing. (A) Significant factors for PEDIS/IDAS grade (3, 4 vs. 1, 2) in diabetic foot ulcers (DFU). (B) Significant factors for 10-item DFI score (cutoff=20) in DFU. PEDIS, perfusion, extent, depth, infection and sensation; IDAS, Infectious Diseases Society of America; DFI, diabetic foot infection; GFR, glomerular filtration rate; WBC, white blood cell; OR, odds ratio. 


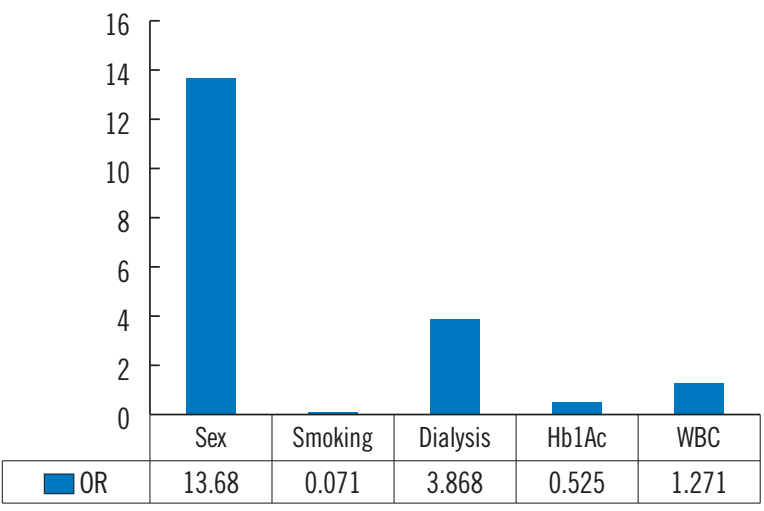

Fig. 3. Bacterial culture (Gram-aerobic vs. others) in DFU. Multivariate binary logistic regressing. DFU, diabetic foot ulcers; $\mathrm{HbA1c}$, glycosylated hemoglobin; WBC, white blood cell; OR, odds ratio.

tients with high WBC and osteomyelitis might already have an infection, which is likely to lead to greater severity of disease.

To the best of our knowledge, there have been no studies analyzing the factors that influence bacterial culture test results, which are the basis of antibiotic therapy in diabetic foot ulcers patients. In our study, sex (male), dialysis, and high WBC seem to affect the possibility of Gram-negative aerobic bacterial infection more than other bacterial infections (Fig. 3).

Polymicrobial organisms have been isolated from infected diabetic foot ulcer patients since the 1980s. Gram-positive cocci bacteria are the most commonly isolated microorganisms, Gram-negative being the second, and anaerobic bacteria being the third. These results do not reflect the various clinical features and can vary from region to region, which may affect the treatment strategy.

Despite the previous results, according to the literature from Asia and the Middle East, the most predominant bacteria isolated from diabetic foot ulcers patients are said to be Gramnegative organisms [25]. Similar to previous studies, our study has shown that Gram-negative aerobic bacteria was the most predominant isolated organism. The possibility of a variance in results of bacterial culture tests in diabetic foot ulcers patients across different regions and races must be noted, and custom treatment plans for each region and/or race should be considered.

Our study had some limitations. First, this study was based on a retrospective chart review. Second, the iagnosis of osteomyelitis was made only in a limited number of patients, using three-phase bone scan. Though three-phase bone scans are less sensitive and specific than magnetic resonance imaging, they were used for rapid screening at an appropriate point in time.

In conclusion, the present study has confirmed significant factors affecting vascular status, wound status, and bacterial culture tests, including co-morbidities, as well as foot and ulcer characteristics at baseline. Older age and decreased GFR have affected both the vascular status and wound status. In addition, sex (male), dialysis, and high WBC seem to affect the possibility of Gram-negative aerobic bacterial infection more than other bacterial infections. However, recent studies have shown that empirical treatments may vary from region to region and that further research is needed, as the predominant bacteria tends to differ accross different ethnic groups and regions.

\section{Conflict of interest}

No potential conflicts of interest relevant to this article are reported.

\section{Acknowledgments}

Jong-Lim Kim https://orcid.org/0000-0002-7110-4574

Jin Yong Shin https://orcid.org/0000-0003-2680-7411

Si-Gyun Roh https://orcid.org/0000-0003-2865-0075

Suk Choo Chang https://orcid.org/0000-0003-0679-182X

Nae-Ho Lee https://orcid.org/0000-0003-1354-8203

\section{References}

1. Ince P, Kendrick D, Game F, et al. The association between baseline characteristics and the outcome of foot lesions in a UK population with diabetes. Diabet Med 2007;24:977-81.

2. Prompers L, Huijberts M, Schaper N, et al. Resource utilisation and costs associated with the treatment of diabetic foot ulcers: prospective data from the Eurodiale study. Diabetologia 2008;51:1826-34.

3. Prompers L, Schaper N, Apelqvist J, et al. Prediction of outcome in individuals with diabetic foot ulcers: focus on the differences between individuals with and without peripheral arterial disease. The EURODIALE study. Diabetologia 2008; 51:747-55.

4. Beckert S, Witte M, Wicke C, et al. A new wound-based severity score for diabetic foot ulcers: a prospective analysis of 1,000 patients. Diabetes Care 2006;29:988-92.

5. Miyajima S, Shirai A, Yamamoto S, et al. Risk factors for major limb amputations in diabetic foot gangrene patients. Dia- 
betes Res Clin Pract 2006;71:272-9.

6. Cardoso CR, Salles GF. Macro and microvascular complications are determinants of increased infection-related mortality in Brazilian type 2 diabetes mellitus patients. Diabetes Res Clin Pract 2007;75:51-8.

7. Lipsky BA, Polis AB, Lantz KC, et al. The value of a wound score for diabetic foot infections in predicting treatment outcome: a prospective analysis from the SIDESTEP trial. Wound Repair Regen 2009;17:671-7.

8. Hinchliffe RJ, Andros G, Apelqvist J, et al. A systematic review of the effectiveness of revascularization of the ulcerated foot in patients with diabetes and peripheral arterial disease. Diabetes Metab Res Rev 2012;28 Suppl 1:179-217.

9. Varela C, Acin F, de Haro J, et al. The role of foot collateral vessels on ulcer healing and limb salvage after successful endovascular and surgical distal procedures according to an angiosome model. Vasc Endovascular Surg 2010;44:65460.

10. Awad S, Karkos CD, Serrachino-Inglott F, et al. The impact of diabetes on current revascularisation practice and clinical outcome in patients with critical lower limb ischaemia. Eur J Vasc Endovasc Surg 2006;32:51-9.

11. Forsythe RO, Brownrigg J, Hinchliffe RJ. Peripheral arterial disease and revascularization of the diabetic foot. Diabetes Obes Metab 2015;17:435-44.

12. Moulik PK, Mtonga R, Gill GV. Amputation and mortality in new-onset diabetic foot ulcers stratified by etiology. Diabetes Care 2003;26:491-4.

13. Nirantharakumar K, Saeed M, Wilson I, et al. In-hospital mortality and length of stay in patients with diabetes having foot disease. J Diabetes Complications 2013;27:454-8.

14. Nather A, Bee CS, Huak CY, et al. Epidemiology of diabetic foot problems and predictive factors for limb loss. J Diabetes Complications 2008;22:77-82.

15. Aiello A, Anichini R, Brocco E, et al. Treatment of peripheral arterial disease in diabetes: a consensus of the Italian Societies of Diabetes (SID, AMD), Radiology (SIRM) and Vascular Endovascular Surgery (SICVE). Nutr Metab Cardiovasc
Dis 2014:24:355-69.

16. Garimella PS, Hart PD, O'Hare A, et al. Peripheral artery disease and CKD: a focus on peripheral artery disease as a critical component of CKD care. Am J Kidney Dis 2012;60: 641-54.

17. Albers M, Romiti M, De Luccia N, et al. An updated metaanalysis of infrainguinal arterial reconstruction in patients with end-stage renal disease. J Vasc Surg 2007;45:536-42.

18. Reddan DN, Marcus RJ, Owen WF Jr, et al. Long-term outcomes of revascularization for peripheral vascular disease in end-stage renal disease patients. Am J Kidney Dis 2001;38: 57-63.

19. Ramdev P, Rayan SS, Sheahan M, et al. A decade experience with infrainguinal revascularization in a dialysis-dependent patient population. J Vasc Surg 2002;36:969-74.

20. O'Hare AM, Sidawy AN, Feinglass J, et al. Influence of renal insufficiency on limb loss and mortality after initial lower extremity surgical revascularization. J Vasc Surg 2004;39:70916.

21. Selvin E, Marinopoulos S, Berkenblit G, et al. Meta-analysis: glycosylated hemoglobin and cardiovascular disease in diabetes mellitus. Ann Intern Med 2004;141:421-31.

22. Stratton IM, Adler Al, Neil HA, et al. Association of glycaemia with macrovascular and microvascular complications of type 2 diabetes (UKPDS 35): prospective observational study. BMJ 2000;321:405-12.

23. Selvin E, Coresh J, Golden SH, et al. Glycemic control and coronary heart disease risk in persons with and without diabetes: the atherosclerosis risk in communities study. Arch Intern Med 2005;165:1910-6.

24. Selvin E, Wattanakit K, Steffes MW, et al. HbAlc and peripheral arterial disease in diabetes: the Atherosclerosis Risk in Communities study. Diabetes Care 2006;29:877-82.

25. Ramakant $P$, Verma AK, Misra R, et al. Changing microbiological profile of pathogenic bacteria in diabetic foot infections: time for a rethink on which empirical therapy to choose? Diabetologia 2011;54:58-64. 